\title{
CONDITIONAL GENERALIZED FOURIER-FEYNMAN TRANSFORM OF FUNCTIONALS IN A FRESNEL TYPE CLASS
}

\author{
Seung Jun Chang
}

\begin{abstract}
In this paper we define the concept of a conditional generalized Fourier-Feynman transform on very general function space $C_{a, b}[0, T]$. We then establish the existence of the conditional generalized FourierFeynman transform for functionals in a Fresnel type class. We also obtain several results involving the conditional transform. Finally we present functionals to apply our results. The functionals arise naturally in Feynman integration theories and quantum mechanics.
\end{abstract}

\section{Introduction}

Let $C_{0}[0, T]$ denote one-parameter Wiener space, that is the space of realvalued continuous functions $x(t)$ on $[0, T]$ with $x(0)=0$. In [21], Yeh introduced the concept of a conditional Wiener integral and derived a Fourier inversion formula for changing conditional expectations into nonconditional expectations. In [17], Park and Skoug obtained a very simple formula for expressing conditional Wiener integrals in terms of ordinary Wiener integrals. The authors, in [21] and [17], derived the Kac-Feynman integral equation for time independent potential functions using their own result, respectively. In [2], Chang and Chung studied the conditional function space integrals and related topics on a very general function space $C_{a, b}[0, T]$ using the vector-valued conditioning function

$$
X(x)=\left(x\left(t_{1}\right), \ldots, x\left(t_{n}\right)\right), \quad 0=t_{0}<t_{1}<\cdots<t_{n}=T .
$$

In [10], Chang, Choi and Skoug established a very simple formula for expressing conditional function space integrals in terms of nonconditional function space integrals using a very general conditioning function $X(x)$ on $C_{a, b}[0, T]$ that need not depend upon the values of $X$ at only finitely many points of $(0, T]$

Received April 3, 2010.

2010 Mathematics Subject Classification. Primary 60J65, 28C20.

Key words and phrases. generalized Brownian motion, Fresnel type class, generalized Fourier-Feynman transform, conditional generalized Feynman integral, conditional generalized Fourier-Feynman transform.

The present research was conducted by the research fund of Dankook University in 2009. 
like in the equation (1.1) above; see the equation (4.2) below. The function space $C_{a, b}[0, T]$ induced by generalized Brownian motion was introduced by J. Yeh in [19] and was used extensively by Chang and Chung [2], Chang and Skoug [4], Chang, Choi and Skoug [7-9] and Chang, Chung and Skoug [11].

In [14], Chung and Skoug introduced the concept of a conditional Feynman integral and applied their results to obtain a fundamental solution of Schrödinger equation, whereas in [18], Park and Skoug introduced the concept of a conditional Fourier-Feynman transform on Wiener space. Other work involving conditional Feynman integrals and conditional Fourier-Feynman transforms on Wiener space include [3, 13]. In [8], Chang, Choi and Skoug established various integration by parts formulas for conditional generalized Feynman integrals and conditional generalized Fourier-Feynman transforms(CGFFT) using the conditioning function $X(x)=x(T), x \in C_{a, b}[0, T]$.

In this paper, working in the setting of general function space $C_{a, b}[0, T]$ and using the conditioning function $X$ given by the equation (4.2) below, we introduce a concept of a CGFFT and obtain several results for the CGFFT of functionals in a Fresnel type class.

The Wiener process used in $[3,13,14,17,18,21]$ is stationary in time and is free of drift while the stochastic process used in $[2,4,7-10,11,19]$ and in this paper is nonstationary in time and is subject to a drift $a(t)$. However when $a(t) \equiv 0$ and $b(t)=t$ on $[0, T], C_{a, b}[0, T]$ reduces to Wiener space $C_{0}[0, T]$.

\section{Preliminaries}

In this section, we briefly list some of the preliminaries from $[4,7,9]$ that we need to establish our results in next sections.

Let $\left(C_{a, b}[0, T], \mathcal{B}\left(C_{a, b}[0, T]\right), \mu\right)$ denote the function space induced by the generalized Brownian motion $Y$ determined by continuous functions $a(t)$ and $b(t)$ where $\mathcal{B}\left(C_{a, b}[0, T]\right)$ is the Borel $\sigma$-algebra induced by the sup-norm, see $[19,20]$. We assume in this paper that $a(t)$ is an absolutely continuous realvalued function on $[0, T]$ with $a(0)=0, a^{\prime}(t) \in L^{2}[0, T]$, and $b(t)$ is a strictly increasing, continuously differentiable real-valued function with $b(0)=0$ and $b^{\prime}(t)>0$ for each $t \in[0, T]$. Then we can consider the coordinate process $Z:[0, T] \times C_{a, b}[0, T] \rightarrow \mathbb{R}$ given by $Z(t, x)=x(t)$ which is a continuous version of $Y$ [20]. The generalized Brownian motion $Z$ is a Gaussian process with mean function $a(t)$ and covariance function $r(s, t)=\min \{b(s), b(t)\}$.

A subset $B$ of $C_{a, b}[0, T]$ is said to be scale-invariant measurable provided $\rho B$ is $\mathcal{B}\left(C_{a, b}[0, T]\right)$-measurable for all $\rho>0$, and a scale-invariant measurable set $N$ is said to be a scale-invariant null set provided $\mu(\rho N)=0$ for all $\rho>0$. A property that holds except on a scale-invariant null set is said to hold scaleinvariant almost everywhere(s-a.e.). If two functionals $F$ and $G$ defined on $C_{a, b}[0, T]$ are equal s-a.e., then we write $F \approx G$.

Let $L_{a, b}^{2}[0, T]$ be the space of functions on $[0, T]$ which are Lebesgue measurable and square integrable with respect to the Lebesgue-Stieltjes measures 
on $[0, T]$ induced by $a(t)$ and $b(t)$ : i.e.,

$$
L_{a, b}^{2}[0, T]=\left\{v: \int_{0}^{T} v^{2}(s) d b(s)<\infty \text { and } \int_{0}^{T} v^{2}(s) d|a|(s)<\infty\right\},
$$

where $|a|(t)$ is the total variation function of $a(t)$. Then $L_{a, b}^{2}[0, T]$ is a separable Hilbert space with inner product defined by

$$
(u, v)_{a, b}=\int_{0}^{T} u(t) v(t) d[b(t)+|a|(t)] .
$$

In particular, note that $\|u\|_{a, b}=0$ if and only if $u(t)=0$ for $m_{L}$-a.e. on $[0, T]$ where $m_{L}$ is the Lebesgue measure on $[0, T]$. Also note that if $a(t) \equiv 0$ and $b(t)=t$, then $L_{a, b}^{2}[0, T]=L^{2}[0, T]$. In fact,

$$
\left(L_{a, b}^{2}[0, T],\|\cdot\|_{a, b}\right) \subset\left(L_{0, b}^{2}[0, T],\|\cdot\|_{0, b}\right)=\left(L^{2}[0, T],\|\cdot\|_{2}\right)
$$

since the two norms $\|\cdot\|_{0, b}$ and $\|\cdot\|_{2}$ are equivalent.

For each $v \in L_{a, b}^{2}[0, T]$, the Paley-Wiener-Zygmund(PWZ) stochastic integral

$$
\langle v, x\rangle=\lim _{n \rightarrow \infty} \int_{0}^{T} \sum_{j=1}^{n}\left(v, \phi_{j}\right)_{a, b} \phi_{j}(t) d x(t)
$$

exists for $\mu$-a.e. $x \in C_{a, b}[0, T]$, where $\left\{\phi_{j}\right\}_{j=1}^{\infty}$ is a complete orthonormal set of real-valued functions of bounded variation on $[0, T]$ such that $\left(\phi_{j}, \phi_{k}\right)_{a, b}=\delta_{j k}$ (the Kronecker delta). If $v$ is of bounded variation on $[0, T]$, then the PWZ stochastic integral $\langle v, x\rangle$ equals the Riemann-Stieltjes integral $\int_{0}^{T} v(t) d x(t)$ for s-a.e. $x \in C_{a, b}[0, T]$.

Remark 2.1. For each $v \in L_{a, b}^{2}[0, T]$, the PWZ stochastic integral $\langle v, x\rangle$ is a Gaussian random variable on $C_{a, b}[0, T]$ with mean $\int_{0}^{T} v(s) d a(s)$ and variance $\int_{0}^{T} v^{2}(s) d b(s)$. Note that for all $u, v \in L_{a, b}^{2}[0, T]$,

$$
\int_{C_{a, b}[0, T]}\langle u, x\rangle\langle v, x\rangle d \mu(x)=\int_{0}^{T} u(s) v(s) d b(s)+\int_{0}^{T} u(s) d a(s) \int_{0}^{T} v(s) d a(s) .
$$

Hence we see that for all $u, v \in L_{a, b}^{2}[0, T], \int_{0}^{T} u(s) v(s) d b(s)=0$ if and only if $\langle u, x\rangle$ and $\langle v, x\rangle$ are independent random variables.

Let

$$
C_{a, b}^{\prime}[0, T]=\left\{w \in C_{a, b}[0, T]: w(t)=\int_{0}^{t} z(s) d b(s) \text { for some } z \in L_{a, b}^{2}[0, T]\right\} .
$$

For $w \in C_{a, b}^{\prime}[0, T]$, with $w(t)=\int_{0}^{t} z(s) d b(s)$ for $t \in[0, T]$, let $D_{t}: C_{a, b}^{\prime}[0, T] \rightarrow$ $L_{a, b}^{2}[0, T]$ be defined by the formula

$$
D_{t} w=z(t)=\frac{w^{\prime}(t)}{b^{\prime}(t)} .
$$


Then $C_{a, b}^{\prime} \equiv C_{a, b}^{\prime}[0, T]$ with inner product

$$
\left(w_{1}, w_{2}\right)_{C_{a, b}^{\prime}}=\int_{0}^{T} D_{t} w_{1} D_{t} w_{2} d b(t)=\int_{0}^{T} z_{1}(t) z_{2}(t) d b(t)
$$

is a separable Hilbert space. For more details, see $[9,12]$.

Note that the two separable Hilbert spaces $L_{a, b}^{2}[0, T]$ and $C_{a, b}^{\prime}[0, T]$ are homeomorphic under the linear operator given by the equation (2.1).

Throughout this paper, we assume $a \in C_{a, b}^{\prime}[0, T]$ and for notational convenience we will use the notation $(w, x)^{\sim}$ instead of $\left\langle D_{t} w, x\right\rangle$. Then we have the following assertions.

(1) For each $w \in C_{a, b}^{\prime}[0, T]$, the random variable $x \mapsto(w, x)^{\sim}$ is Gaussian with mean $(w, a)_{C_{a, b}^{\prime}}$ and variance $\|w\|_{C_{a, b}^{\prime}}^{2}$.

(2) $(w, \alpha x)^{\sim}=\alpha(w, x)^{\sim}=(\alpha w, x)^{\sim}$ for any real number $\alpha, w \in C_{a, b}^{\prime}[0, T]$ and $x \in C_{a, b}[0, T]$.

(3) If $\left\{w_{1}, \ldots, w_{n}\right\}$ is an orthonormal set in $C_{a, b}^{\prime}[0, T]$, then the random variables $\left(w_{i}, x\right)^{\sim}$ 's are independent.

We denote the function space integral of a $\mathcal{B}\left(C_{a, b}[0, T]\right)$-measurable functional $F$ by

$$
E[F] \equiv E_{x}[F(x)]=\int_{C_{a, b}[0, T]} F(x) d \mu(x)
$$

whenever the integral exists.

Throughout this paper, let $\mathbb{C}, \mathbb{C}_{+}$and $\tilde{\mathbb{C}}_{+}$denote the complex numbers, the complex numbers with positive real part, and the nonzero complex numbers with nonnegative real part, respectively. For each $\lambda \in \tilde{\mathbb{C}}_{+}, \lambda^{-1 / 2}\left(\right.$ or $\left.\lambda^{1 / 2}\right)$ is always chosen to have positive real part.

\section{A Fresnel type class $\mathcal{F}\left(C_{a, b}[0, T]\right)$}

We first introduce a Banach algebra of functionals on $C_{a, b}[0, T]$ like a Fresnel class of an abstract Wiener space.

Let $\mathcal{M}\left(C_{a, b}^{\prime}[0, T]\right)$ be the space of $\mathbb{C}$-valued, countably additive (and hence finite) Borel measures on $C_{a, b}^{\prime}[0, T] . \mathcal{M}\left(C_{a, b}^{\prime}[0, T]\right)$ is a Banach algebra under the total variation norm and with convolution as multiplication.

The Fresnel type class $\mathcal{F}\left(C_{a, b}[0, T]\right)$ of functionals on $C_{a, b}[0, T]$ is defined as the space of all stochastic Fourier transforms of elements of $\mathcal{M}\left(C_{a, b}^{\prime}[0, T]\right)$; that is, $F \in \mathcal{F}\left(C_{a, b}[0, T]\right)$ if and only if there exists a measure $f$ in $\mathcal{M}\left(C_{a, b}^{\prime}[0, T]\right)$ such that

$$
F(x)=\int_{C_{a, b}^{\prime}[0, T]} \exp \left\{i(w, x)^{\sim}\right\} d f(w)
$$

for s-a.e. $x \in C_{a, b}[0, T]$. More precisely, since we shall identify functionals which coincide s-a.e. on $C_{a, b}[0, T], \mathcal{F}\left(C_{a, b}[0, T]\right)$ can be regarded as the space of all s-equivalence classes of functionals of the form (3.1). 
The Fresnel type class $\mathcal{F}\left(C_{a, b}[0, T]\right)$ is a Banach algebra with norm

$$
\|F\|=\|f\|=\int_{C_{a, b}^{\prime}[0, T]} d|f|(w) .
$$

In fact, the correspondence $f \mapsto F$ is injective, carries convolution into pointwise multiplication and is a Banach algebra isomorphism where $f$ and $F$ are related by (3.1).

We adopt the definitions and notations of $[4,7]$ for the concept of the generalized Feynman integral and the generalized Fourier-Feynman transform(GFFT) of functionals on $C_{a, b}[0, T]$.

Remark 3.1. In [4] Chang and Skoug introduced a Banach algebra $\mathcal{S}\left(L_{a, b}^{2}[0, T]\right)$ of functionals on $C_{a, b}[0, T]$ given by

$$
\mathcal{S}\left(L_{a, b}^{2}[0, T]\right)=\left\{F: F(x) \approx \int_{L_{a, b}^{2}[0, T]} \exp \{i\langle v, x\rangle\} d \sigma(v), \sigma \in \mathcal{M}\left(L_{a, b}^{2}[0, T]\right)\right\}
$$

and then showed that the generalized Feynman integral and the GFFT exist for functionals in $\mathcal{S}\left(L_{a, b}^{2}[0, T]\right)$ under appropriate conditions.

When $a(t) \equiv 0$ and $b(t)=t$ on $[0, T], \mathcal{S}\left(L_{a, b}^{2}[0, T]\right)$ reduces to the Banach algebra $\mathcal{S}$ introduced by Cameron and Storvick [1]. For more detailed studies of Banach algebras of functionals on classical and abstract Wiener spaces, see [16, pp. 609-629]. Also, for a detailed study of functionals in $\mathcal{F}\left(C_{a, b}[0, T]\right)$, see $[12]$.

For a positive real number $q_{0}$ and $w \in C_{a, b}^{\prime}[0, T]$, let

$$
k\left(q_{0} ; w\right)=\exp \left\{\left(2 q_{0}\right)^{-1 / 2}\|w\|_{C_{a, b}^{\prime}}\|a\|_{C_{a, b}^{\prime}}\right\}
$$

and let

$$
\Gamma_{q_{0}}=\left\{\lambda \in \tilde{\mathbb{C}}_{+}:\left|\operatorname{Im}\left(\lambda^{-1 / 2}\right)\right|<\left(2 q_{0}\right)^{-1 / 2}\right\} .
$$

Also, for $\lambda \in \tilde{\mathbb{C}}$ and $w \in C_{a, b}^{\prime}[0, T]$, let

$$
\psi(\lambda ; w)=\exp \left\{-\frac{1}{2 \lambda}\|w\|_{C_{a, b}^{\prime}}^{2}+\lambda^{-1 / 2}(w, a)_{C_{a, b}^{\prime}}\right\} .
$$

Then for all $\lambda \in \Gamma_{q_{0}}$,

$$
|\psi(\lambda ; w)| \leq \exp \left\{\left|\operatorname{Im}\left(\lambda^{-1 / 2}\right)\right|\|w\|_{C_{a, b}^{\prime}}\|a\|_{C_{a, b}^{\prime}}\right\}<k\left(q_{0} ; w\right) .
$$

We note that for all real $q$ with $|q|>q_{0},(-i q)^{-1 / 2}=1 / \sqrt{2|q|}+i \operatorname{sign}(q) / \sqrt{2|q|}$ and $-i q \in \Gamma_{q_{0}}$.

For a positive real number $q_{0}$, we define a subclass $\mathcal{F}^{q_{0}}$ of $\mathcal{F}\left(C_{a, b}[0, T]\right)$ by

$$
\mathcal{F}^{q_{0}}=\left\{F \in \mathcal{F}\left(C_{a, b}[0, T]\right): \int_{C_{a, b}^{\prime}[0, T]} k\left(q_{0} ; w\right) d|f|(w)<\infty\right\},
$$

where $f$ and $F$ are related by the equation (3.1). 
Remark 3.2. Note that in case $a(t) \equiv 0$ and $b(t)=t$ on $[0, T]$, the function space $C_{a, b}[0, T]$ reduces to the classical Wiener space $C_{0}[0, T]$ and $(w, a)_{C_{a, b}^{\prime}}=0$ for all $w \in C_{a, b}^{\prime}[0, T]=C_{0}^{\prime}[0, T]$. Hence for all $\lambda \in \tilde{\mathbb{C}}_{+},|\psi(\lambda ; w)| \leq 1$ and for any positive real number $q_{0}, \mathcal{F}^{q_{0}}=\mathcal{F}\left(C_{0}[0, T]\right)$.

We now state a theorem for the GFFT of functionals in $\mathcal{F}\left(C_{a, b}[0, T]\right)$ without proof. One can see similar results in $[4,5,6]$.

Theorem 3.3. Let $q_{0}$ be a positive real number. Let $F \in \mathcal{F}^{q_{0}}$ be given by the equation (3.1). Then for all $p \in[1,2]$ and all real $q$ with $|q|>q_{0}$, the $L_{p}$ analytic GFFT of $F, T_{q}^{(p)}(F)$ exists and is given by the formula

$$
T_{q}^{(p)}(F)(y)=\int_{C_{a, b}^{\prime}[0, T]} \exp \left\{i(w, y)^{\sim}\right\} \psi(-i q ; w) d f(w)
$$

for s-a.e. $y \in C_{a, b}[0, T]$, where $\psi$ is given by (3.3). Furthermore, $T_{q}^{(p)}(F)$ belongs to $\mathcal{F}\left(C_{a, b}[0, T]\right)$.

Corollary 3.4. Let $q_{0}$ and $F$ be as in Theorem 3.3. Then for all real $q$ with $|q|>q_{0}$, the generalized analytic Feynman integral of $F, E^{\operatorname{anf}_{q}}[F]$ exists and is given by the formula

$$
E^{\operatorname{anf}_{q}}[F]=T_{q}^{(1)}(F)(0)=\int_{C_{a, b}^{\prime}[0, T]} \psi(-i q ; w) d f(w)
$$

\section{Conditional generalized Fourier-Feynman transform}

In this section for $F: C_{a, b}[0, T] \rightarrow \mathbb{C}$ and $X: C_{a, b}[0, T] \rightarrow \mathbb{R}^{n}$, we first define the conditional function space integral of $F$ given $X$ which we denote by $E(F \mid X)$. Then, using the conditioning function $X$ given by the equation (4.2) below, we define the conditional generalized Feynman integral $E^{\operatorname{anf}_{q}}(F \mid X)$ and the $\operatorname{CGFFT} T_{q}^{(p)}(F \mid X)$.

Let $X: C_{a, b}[0, T] \rightarrow \mathbb{R}^{n}$ be a $\mathcal{B}\left(C_{a, b}[0, T]\right)$-measurable functional whose probability distribution $\mu_{X}$ is absolutely continuous with respect to Lebesgue measure on $\mathbb{R}^{n}$. Let $F$ be a $\mathbb{C}$-valued $\mu$-integrable functional on $C_{a, b}[0, T]$. Then, the conditional function space integral of $F$ given $X$, denoted by $E(F \mid X)$ $(\vec{\eta})$, is a Lebesgue measurable function of $\vec{\eta}$, unique up to null sets in $\mathbb{R}^{n}$, satisfying the equation

$$
\int_{X^{-1}(B)} F(x) d \mu(x)=\int_{B} E(F \mid X)(\vec{\eta}) d \mu_{X}(\vec{\eta})
$$

for all Borel sets $B$ in $\mathbb{R}^{n}$.

Let $\left\{g_{1}, \ldots, g_{n}\right\}$ be any orthonormal set in $C_{a, b}^{\prime}[0, T]$. We note that the corresponding PWZ stochastic integrals $\left(g_{j}, x\right)^{\sim}, j=1, \ldots, n$, form a set of independent Gaussian random variables on $C_{a, b}[0, T]$. Let $X: C_{a, b}[0, T] \rightarrow \mathbb{R}^{n}$ be the Gaussian random vector defined by

$$
X(x)=\left(\left(g_{1}, x\right)^{\sim}, \ldots,\left(g_{n}, x\right)^{\sim}\right) .
$$


Remark 4.1. We note that the conditioning function $X$ given by the equation (1.1) is the special case of $X$ given by the equation (4.2) with

$$
g_{j}(t)=\left[b\left(t_{j}\right)-b\left(t_{j-1}\right)\right]^{-1 / 2} \int_{0}^{t} \chi_{\left[t_{j-1}, t_{j}\right]}(s) d b(s)
$$

for $j=1, \ldots, n$.

Definition 4.2. Let $X: C_{a, b}[0, T] \rightarrow \mathbb{R}^{n}$ be given by the equation (4.2) and let $F: C_{a, b}[0, T] \rightarrow \mathbb{C}$ be a scale-invariant measurable functional such that the function space integral $E\left[F\left(\lambda^{-1 / 2} \cdot\right)\right]$ exists as a finite number for all $\lambda>0$. For $\lambda>0$ let

$$
J_{\lambda}(\vec{\eta})=E\left(F\left(\lambda^{-1 / 2} \cdot\right) \mid X\left(\lambda^{-1 / 2} \cdot\right)\right)(\vec{\eta})
$$

denote the conditional function space integral of $F\left(\lambda^{-1 / 2} \cdot\right)$ given $X\left(\lambda^{-1 / 2} \cdot\right)$. If for almost all $\vec{\eta} \in \mathbb{R}^{n}$, there exists a function $J_{\lambda}^{*}(\vec{\eta})$, analytic in $\lambda$ on $\mathbb{C}_{+}$such that $J_{\lambda}^{*}(\vec{\eta})=J_{\lambda}(\vec{\eta})$ for all $\lambda>0$, then $J_{\lambda}^{*}(\cdot)$ is defined to be the conditional analytic function space integral of $F$ given $X$ with parameter $\lambda$ and for $\lambda \in \mathbb{C}_{+}$ we write

$$
E^{\mathrm{an}_{\lambda}}(F \mid X)(\vec{\eta})=J_{\lambda}^{*}(\vec{\eta})
$$

If for fixed real $q \neq 0$, the limit

$$
\lim _{\lambda \rightarrow-i q} E^{\mathrm{an}_{\lambda}}(F \mid X)(\vec{\eta})
$$

exists for almost every $\vec{\eta} \in \mathbb{R}^{n}$, where $\lambda \rightarrow-i q$ through $\mathbb{C}_{+}$, we denote the value of this limit by $E^{\operatorname{anf}_{q}}(F \mid X)(\vec{\eta})$ and we call it the conditional generalized analytic Feynman integral of $F$ given $X$ with parameter $q$.

Next we define $[\cdot]: \mathbb{R}^{n} \rightarrow C_{a, b}^{\prime}[0, T]$ by $[\vec{\eta}]=\sum_{j=1}^{n} \eta_{j} g_{j}$ for $\vec{\eta}=\left(\eta_{1}, \ldots, \eta_{n}\right) \in$ $\mathbb{R}^{n}$ and we write

$$
[x] \equiv[X(x)]=\sum_{j=1}^{n}\left(g_{j}, x\right)^{\sim} g_{j}
$$

for $x \in C_{a, b}[0, T]$.

We quote the following theorem from [10] which plays an important role in this paper.

Theorem 4.3. Let $X$ be given by the equation (4.2) and let $F$ be a $\mu$-integrable functional on $C_{a, b}[0, T]$. Then

$$
E(F \mid X)(\vec{\eta})=E_{x}[F(x-[x]+[\vec{\eta}])] .
$$

In view of Theorem 4.3, we can define the CGFFT of functionals on function space $C_{a, b}[0, T]$.

Definition 4.4. For $\lambda \in \mathbb{C}_{+}$and $y \in C_{a, b}[0, T]$, let $T_{\lambda}(F \mid X)(y, \vec{\eta})$ denote the conditional analytic function space integral of $F(y+\cdot)$ given $X(\cdot)$ with parameter $\lambda$; that is to say

$$
\begin{aligned}
T_{\lambda}(F \mid X)(y, \vec{\eta}) & =E^{\mathrm{an}_{\lambda}}(F(y+\cdot) \mid X)(\vec{\eta}) \\
& =E_{x}^{\mathrm{an}_{\lambda}}[F(y+x-[x]+[\vec{\eta}])] .
\end{aligned}
$$


Then for $p \in[1,2]$ we define the CGFFT of $F$ given $X$ by the formula $\left(\lambda \in \mathbb{C}_{+}\right)$,

$$
T_{q}^{(p)}(F \mid X)(y, \vec{\eta})=\left\{\begin{array}{lc}
\lim \operatorname{im}_{\lambda \rightarrow-i q} T_{\lambda}(F \mid X)(y, \vec{\eta}), & 1<p \leq 2 \\
\lim _{\lambda \rightarrow-i q} T_{\lambda}(F \mid X)(y, \vec{\eta}), & p=1
\end{array}\right.
$$

if it exists. In the left hand side of (4.5), the notation 1. i. m. $\lambda_{\lambda \rightarrow-i q}$ means that for each $\rho>0$,

$$
\lim _{\lambda \rightarrow-i q} \int_{C_{a, b}[0, T]}\left|T_{\lambda}(F \mid X)(\rho y, \vec{\eta})-T_{q}^{(p)}(F \mid X)(\rho y, \vec{\eta})\right|^{p^{\prime}} d \mu(y)=0,
$$

where $1 / p+1 / p^{\prime}=1$. Note that in the case $p=1$,

$$
\begin{aligned}
T_{q}^{(1)}(F \mid X)(y, \vec{\eta}) & =E^{\operatorname{anf}_{q}}(F(y+\cdot) \mid X)(\vec{\eta}) \\
& =E_{x}^{\operatorname{anf}_{q}}[F(y+x-[x]+[\vec{\eta}])] .
\end{aligned}
$$

\section{Conditional transforms of functionals in $\mathcal{F}\left(C_{a, b}[0, T]\right)$}

In this section we establish the existence of the CGFFT of functionals in $\mathcal{F}\left(C_{a, b}[0, T]\right)$. We then use the result for the CGFFT to obtain an expression of the GFFT of functionals in $\mathcal{F}\left(C_{a, b}[0, T]\right)$. We also establish a translation theorem for the CGFFT of functionals in $\mathcal{F}\left(C_{a, b}[0, T]\right)$.

Let $\left\{g_{1}, \ldots, g_{n}\right\}$ be an orthonormal set in $C_{a, b}^{\prime}[0, T]$. For each $w \in C_{a, b}^{\prime}[0, T]$, let

$$
p(w)=w-[w] .
$$

Then the PWZ stochastic integral $(p(w), x)^{\sim}$ is a Gaussian random variable with mean

$$
A(p(w))=(w-[w], a)_{C_{a, b}^{\prime}}=(w, a)_{C_{a, b}^{\prime}}-\sum_{j=1}^{n}\left(g_{j}, w\right)_{C_{a, b}^{\prime}}\left(g_{j}, a\right)_{C_{a, b}^{\prime}}
$$

and variance

$$
B(p(w)) \equiv\|w-[w]\|_{C_{a, b}^{\prime}}^{2}=\|w\|_{C_{a, b}^{\prime}}^{2}-\sum_{j=1}^{n}\left(g_{j}, w\right)_{C_{a, b}^{\prime}}^{2} .
$$

The following lemma is useful in establishing our main theorem for the CGFFT of functionals $F$ in $\mathcal{F}\left(C_{a, b}[0, T]\right)$. The proof follows from (4.3), (5.1), (5.2) and the change of variable formula.

Lemma 5.1. Let $X$ be given by the equation (4.2) above. For $w \in C_{a, b}^{\prime}[0, T]$, let $G: C_{a, b}[0, T] \rightarrow \mathbb{C}$ be defined by $G(x)=\exp \left\{i(w, x)^{\sim}\right\}$. Then for $\rho>0$ and $\vec{\eta} \in \mathbb{R}^{n}$,

$$
E(G(\rho \cdot) \mid X(\rho \cdot))(\vec{\eta})=\exp \left\{i(w,[\vec{\eta}])_{C_{a, b}^{\prime}}-\frac{\rho^{2}}{2} B(p(w))+i \rho A(p(w))\right\},
$$

where $A(p(w))$ and $B(p(w))$ are given by the equations (5.1) and (5.2). 
For a positive real number $q_{0}$ and $w \in C_{a, b}^{\prime}[0, T]$, let

$$
k_{n}\left(q_{0} ; w\right)=\exp \left\{(1+n)\left(2 q_{0}\right)^{-1 / 2}\|w\|_{C_{a, b}^{\prime}}\|a\|_{C_{a, b}^{\prime}}\right\}
$$

and for $\lambda \in \tilde{\mathbb{C}}_{+}$let

$$
\Psi(\lambda ; p(w))=\exp \left\{-\frac{1}{2 \lambda} B(p(w))+i \lambda^{-1 / 2} A(p(w))\right\} .
$$

Let $\Gamma_{q_{0}}$ be given by (3.2). Then for all $\lambda \in \Gamma_{q_{0}}$,

$$
\begin{aligned}
& |\Psi(\lambda ; p(w))| \\
\leq & \exp \left\{\left|\operatorname{Im}\left(\lambda^{-1 / 2}\right)\right||A(p(w))|\right\} \\
< & \exp \left\{\left(2 q_{0}\right)^{-1 / 2}\left(\|w\|_{C_{a, b}^{\prime}}\|a\|_{C_{a, b}^{\prime}}+\sum_{j=1}^{n}\|w\|_{C_{a, b}^{\prime}}\left\|g_{j}\right\|_{C_{a, b}^{\prime}}^{2}\|a\|_{C_{a, b}^{\prime}}\right)\right\} \\
= & k_{n}\left(q_{0} ; w\right) .
\end{aligned}
$$

To obtain our results for the CGFFT, for positive real number $q_{0}$, we define a subclass $\mathcal{F}_{n}^{q_{0}}$ of $\mathcal{F}\left(C_{a, b}[0, T]\right)$ by

$$
\mathcal{F}_{n}^{q_{0}}=\left\{F \in \mathcal{F}\left(C_{a, b}[0, T]\right): \int_{C_{a, b}^{\prime}[0, T]} k_{n}\left(q_{0} ; w\right) d|f|(w)<\infty\right\},
$$

where $f$ and $F$ are related by the equation (3.1).

Remark 5.2. (i) $\mathcal{F}_{n}^{q_{0}}$ is a subclass of $\mathcal{F}^{q_{0}}$ for each positive real number $q_{0}$ and every $n \in \mathbb{N}$.

(ii) When $a(t) \equiv 0$ and $b(t)=t$ on $[0, T], A(p(w))=0$ for all $w \in$ $C_{a, b}^{\prime}[0, T]=C_{0}^{\prime}[0, T]$. Hence for all $\lambda \in \tilde{\mathbb{C}}_{+},|\Psi(\lambda ; p(w))| \leq 1$ and for any positive real number $q_{0}, \mathcal{F}_{n}^{q_{0}}=\mathcal{F}\left(C_{0}[0, T]\right)$.

In next theorem, we establish the existence of the CGFFT of functionals in $\mathcal{F}\left(C_{a, b}[0, T]\right)$.

Theorem 5.3. Let $X$ be given by the equation (4.2) above and let $q_{0}$ be a positive real number. Let $F \in \mathcal{F}_{n}^{q_{0}}$ be given by the equation (3.1). Then for all $p \in[1,2]$ and all real $q$ with $|q|>q_{0}$, the CGFFT of $F, T_{q}^{(p)}(F \mid X)$ exists and is given by the formula

$$
\begin{aligned}
& T_{q}^{(p)}(F \mid X)(y, \vec{\eta}) \\
= & \int_{C_{a, b}^{\prime}[0, T]} \exp \left\{i(w, y)^{\sim}+i(w,[\vec{\eta}])_{C_{a, b}^{\prime}}\right\} \Psi(-i q ; p(w)) d f(w)
\end{aligned}
$$

for s-a.e. $y \in C_{a, b}[0, T]$, where $\Psi$ is given by the equation (5.5). 
Proof. Using (3.1), (4.4), the Fubini theorem, (5.3) and (5.5), we obtain that for $\lambda>0$ and $\vec{\eta} \in \mathbb{R}^{n}$,

$$
\begin{aligned}
& E\left(F\left(y+\lambda^{-1 / 2} \cdot\right) \mid X\left(\lambda^{-1 / 2} \cdot\right)\right)(\vec{\eta}) \\
= & E\left(\int_{C_{a, b}^{\prime}[0, T]} \exp \left\{i\left(w, y+\lambda^{-1 / 2} \cdot\right)^{\sim}\right\} d f(w) \mid X\left(\lambda^{-1 / 2} \cdot\right)\right)(\vec{\eta}) \\
= & \int_{C_{a, b}^{\prime}[0, T]} \exp \left\{i(w, y)^{\sim}\right\} E\left(\exp \left\{i\left(w, \lambda^{-1 / 2} \cdot\right)^{\sim}\right\} \mid X\left(\lambda^{-1 / 2} \cdot\right)\right)(\vec{\eta}) d f(w) \\
= & \int_{C_{a, b}^{\prime}[0, T]} \exp \left\{i(w, y)^{\sim}+i(w,[\vec{\eta}])_{C_{a, b}^{\prime}}\right\} \Psi(\lambda ; p(w)) d f(w) .
\end{aligned}
$$

From this and Definition 4.4, and by a careful examination, we can see that:

(i) $T_{\lambda}(F \mid X)(y, \vec{\eta})=E\left(F\left(y+\lambda^{-1 / 2} \cdot\right) \mid X\left(\lambda^{-1 / 2} \cdot\right)\right)(\vec{\eta})$ is an analytic function of $\lambda$ throughout the domain $\operatorname{Int}\left(\Gamma_{q_{0}}\right)$, where $\Gamma_{q_{0}}$ is given by (3.2);

(ii) $T_{q}^{(1)}(F \mid X)(y, \vec{\eta})=\lim _{\lambda \rightarrow-i q} T_{\lambda}(F \mid X)(y, \vec{\eta})$ exists for s-a.e. $y \in C_{a, b}[0, T]$ and $\vec{\eta} \in \mathbb{R}^{n}$, and is given by the equation (5.6) above; and

(iii) for $p \in(1,2], T_{q}^{(p)}(F \mid X)(y, \vec{\eta})=$ l.i.m. ${ }_{\lambda \rightarrow-i q} T_{\lambda}(F \mid X)(y, \vec{\eta})$ exists for s-a.e. $y \in C_{a, b}[0, T]$ and $\vec{\eta} \in \mathbb{R}^{n}$, and is given by the equation (5.6) above.

In evaluations of $\lim _{\lambda \rightarrow-i q} T_{\lambda}(F \mid X)(y, \vec{\eta})$ and l.i.m. $\lambda_{\lambda \rightarrow-i q} T_{\lambda}(F \mid X)(y, \vec{\eta})$, the dominating functions are given by $k_{n}\left(q_{0} ; w\right)$ and $\left(2 \int_{C_{a, b}^{\prime}[0, T]} k_{n}\left(q_{0} ; w\right) d|f|(w)\right)^{p^{\prime}}$, respectively.

The following corollary follows from (5.6) with $p=1$ and (4.6).

Corollary 5.4. Let $X, q_{0}$ and $F$ be as in Theorem 5.3. Then for all real $q$ with $|q|>q_{0}$, the conditional generalized Feynman integral of $F, E^{\operatorname{anf}_{q}}(F \mid X)$ exists and is given by the formula

$$
\begin{aligned}
E^{\operatorname{anf}_{q}(F \mid X)(\vec{\eta})} & =T_{q}^{(1)}(F \mid X)(0, \vec{\eta}) \\
& =\int_{C_{a, b}^{\prime}[0, T]} \exp \left\{i(w,[\vec{\eta}])_{C_{a, b}^{\prime}}\right\} \Psi(-i q ; p(w)) d f(w),
\end{aligned}
$$

where $\Psi$ is given by the equation (5.5).

In our next theorem, by using the techniques of similar to those used in [14], we show that if we multiply $T_{q}^{(p)}(F \mid X)(y, \vec{\eta})$ by

$$
\varpi(-i q ; \vec{\eta}) \equiv\left(\frac{-i q}{2 \pi}\right)^{\frac{n}{2}} \exp \left\{\frac{i q}{2} \sum_{j=1}^{n}\left[\eta_{j}-(-i q)^{-1 / 2}\left(g_{j}, a\right)_{C_{a, b}^{\prime}}\right]^{2}\right\}
$$

the analytic extension of the Radon-Nykodym derivative evaluated at $\lambda=-i q$, and then integrate over $\mathbb{R}^{n}$ we obtain the $\operatorname{GFFT} T_{q}^{(p)}(F)(y)$. However, to do 
so we also need the following summation procedure as in [15, p. 340]. Let

$$
\int_{\mathbb{R}^{n}} f(\vec{\eta}) d \vec{\eta}=\lim _{M \rightarrow \infty} \int_{\mathbb{R}^{n}} f(\vec{\eta}) \exp \left\{-\left(\sum_{j=1}^{n} \eta_{j}^{2}\right) / 2 M\right\} d \vec{\eta}
$$

whenever the expression on the right-hand side exists. But if $f \in L^{1}\left(\mathbb{R}^{n}\right)$, it is clear by the dominated convergence theorem that

$$
\int_{\mathbb{R}^{n}} f(\vec{\eta}) d \vec{\eta}=\int_{\mathbb{R}^{n}} f(\vec{\eta}) d \vec{\eta} .
$$

To establish the equation (5.10) below, the following well-known integration formula is useful:

$$
\int_{\mathbb{R}} \exp \left\{-\alpha u^{2}+\beta u\right\} d u=\sqrt{\frac{\pi}{\alpha}} \exp \left\{\frac{\beta^{2}}{4 \alpha}\right\}
$$

for complex numbers $\alpha$ and $\beta$ with $\operatorname{Re}(\alpha)>0$.

Theorem 5.5. Let $X, q_{0}$ and $F$ be as in Theorem 5.3. Then for all $p \in[1,2]$ and all real $q$ with $|q|>q_{0}$,

$$
T_{q}^{(p)}(F)(y)=\overline{\int_{\mathbb{R}^{n}}} T_{q}^{(p)}(F \mid X)(y, \vec{\eta}) \varpi(-i q ; \vec{\eta}) d \vec{\eta}
$$

for s-a.e. $y \in C_{a, b}[0, T]$, where $\varpi$ is given by $(5.7)$.

Proof. Since $\mathcal{F}_{n}^{q_{0}} \subset \mathcal{F}^{q_{0}}$, the GFFT $T_{q}^{(p)}(F)$ and the CGFFT $T_{q}^{(p)}(F \mid X)$ of $F$ exist for all $p \in[1,2]$ by Theorems 3.3 and 5.3, respectively. Thus we need only to verify the equality in equation (5.10).

Let $q$ be a nonzero real number with $|q|>q_{0}$. By using (5.6), (5.8), the Fubini theorem, (5.9) and a simple calculation, we obtain that

$$
\begin{aligned}
& \overline{\int_{\mathbb{R}^{n}}} T_{q}^{(p)}(F \mid X)(y, \vec{\eta}) \varpi(-i q ; \vec{\eta}) d \vec{\eta} \\
= & \lim _{M \rightarrow \infty} \int_{\mathbb{R}^{n}} T_{q}^{(p)}(F \mid X)(y, \vec{\eta}) \varpi(-i q ; \vec{\eta}) \exp \left\{-\sum_{j=1}^{n} \frac{\eta_{j}^{2}}{2 M}\right\} d \vec{\eta} \\
(5.11)= & \lim _{M \rightarrow \infty} \int_{C_{a, b}^{\prime}[0, T]} \exp \left\{i(w, y)^{\sim}\right\} \Psi(-i q ; p(w)) \\
& {\left[\int_{\mathbb{R}^{n}} \varpi(-i q ; \vec{\eta}) \times \exp \left\{-\sum_{j=1}^{n} \frac{\eta_{j}^{2}}{2 M}+i \sum_{j=1}^{n}\left(g_{j}, w\right)_{\left.C_{a, b}^{\prime} \eta_{j}\right\}}\right\} \eta_{j}\right] d f(w) } \\
= & \lim _{M \rightarrow \infty} \int_{C_{a, b}^{\prime}[0, T]}\left(\frac{-i q}{2 \pi} \frac{2 \pi M}{1-i q M}\right)^{\frac{n}{2}} \exp \left\{i(w, y)^{\sim}\right\} \Psi(-i q ; p(w))
\end{aligned}
$$




$$
\begin{aligned}
& \times \exp \left\{-\sum_{j=1}^{n} \frac{\left(g_{j}, a\right)_{C_{a, b}^{\prime}}^{2}}{2}-\frac{M}{2(1-i q M)}\right. \\
& \left.\times \sum_{j=1}^{n}\left[\left(g_{j}, w\right)_{C_{a, b}^{\prime}}^{2}-2 q(-i q)^{-1 / 2}\left(g_{j}, w\right)_{C_{a, b}^{\prime}}\left(g_{j}, a\right)_{C_{a, b}^{\prime}}+i q\left(g_{j}, a\right)_{C_{a, b}^{\prime}}^{2}\right]\right\} d f(w) .
\end{aligned}
$$

But a long and tedious calculation shows that for a sufficiently large $M>0$,

$$
\begin{aligned}
& \mid \exp \left\{i(w, y)^{\sim}\right\} \Psi(-i q ; p(w)) \exp \left\{-\sum_{j=1}^{n} \frac{\left(g_{j}, a\right)_{C_{a, b}^{\prime}}^{2}}{2}-\frac{M}{2(1-i q M)}\right. \\
& \left.\quad \times \sum_{j=1}^{n}\left[\left(g_{j}, w\right)_{C_{a, b}^{\prime}}^{2}-2 q(-i q)^{-1 / 2}\left(g_{j}, w\right)_{C_{a, b}^{\prime}}\left(g_{j}, a\right)_{C_{a, b}^{\prime}}+i q\left(g_{j}, a\right)_{C_{a, b}^{\prime}}^{2}\right]\right\} \mid
\end{aligned}
$$

is dominated by $k_{n}\left(q_{0} ; w\right)$.

Using (5.11), the dominated convergence theorem, (5.5), (5.1), (5.2), (3.3) and (3.4), we have the equation (5.10).

The following corollary follows from (5.10) with $p=1$ and (4.6).

Corollary 5.6. Let $X, q_{0}$ and $F$ be as in Theorem 5.3. Then for all real $q$ with $|q|>q_{0}$,

$$
E^{\operatorname{anf}_{q}}[F]=T_{q}^{(1)}(F)(0)=\bar{\int}_{\mathbb{R}^{n}} E^{\operatorname{anf}_{q}}(F \mid X)(\vec{\eta}) \varpi(-i q ; \vec{\eta}) d \vec{\eta},
$$

where $\varpi$ is given by the equation (5.7).

Remark 5.7. (i) Let $x_{0} \in C_{a, b}^{\prime}[0, T]$ and for each nonzero real number $q$, let $\delta_{-q x_{0}} \in \mathcal{M}\left(C_{a, b}^{\prime}[0, T]\right)$ be the Dirac measure concentrated at $-q x_{0}$. Then the functional $H_{-q x_{0}}(x)=\exp \left\{-i q\left(x_{0}, x\right)^{\sim}\right\}$ is an element of $\mathcal{F}\left(C_{a, b}[0, T]\right)$, because

$$
H_{-q x_{0}}(x)=\int_{C_{a, b}^{\prime}[0, T]} \exp \left\{i(w, x)^{\sim}\right\} d \delta_{-q x_{0}}(w) .
$$

Clearly, $H_{-q x_{0}} \in \mathcal{F}_{n}^{r}$ for all positive real number $r$.

For each $F \in \mathcal{F}\left(C_{a, b}[0, T]\right)$, let

$$
F^{*}(x)=F(x) \exp \left\{-i q\left(x_{0}, x\right)^{\sim}\right\} .
$$

Since $\mathcal{F}\left(C_{a, b}[0, T]\right)$ is a Banach algebra, $F^{*}$ is an element of $\mathcal{F}\left(C_{a, b}[0, T]\right)$.

(ii) Using the equation (3.1), we can write $F^{*}(x)$ as follows:

$$
\begin{aligned}
F^{*}(x) & =\int_{C_{a, b}^{\prime}[0, T]} \exp \left\{i\left(w-q x_{0}, x\right)^{\sim}\right\} d f(w) \\
& =\int_{C_{a, b}^{\prime}[0, T]} \exp \left\{i(h, x)^{\sim}\right\} d f_{q x_{0}}^{*}(h),
\end{aligned}
$$

where $f_{q x_{0}}^{*}$ is a measure in $\mathcal{M}\left(C_{a, b}^{\prime}[0, T]\right)$ such that $f_{q x_{0}}^{*}(E) \equiv f\left(E+q x_{0}\right)$ for $E \in \mathcal{B}\left(C_{a, b}^{\prime}[0, T]\right)$. 
Let $F \in \mathcal{F}_{n}^{q_{0}}$ be given by the equation (3.1) and let $F^{*}$ be given by the equation (5.12) with $|q|>q_{0}$. Then

$$
\begin{aligned}
& \int_{C_{a, b}^{\prime}[0, T]} k_{n}\left(q_{0} ; h\right) d\left|f_{q x_{0}}^{*}\right|(h) \\
= & \int_{C_{a, b}^{\prime}[0, T]} k_{n}\left(q_{0} ; w-q x_{0}\right) d|f|(w) \\
\leq & \int_{C_{a, b}^{\prime}[0, T]} \exp \left\{\frac{(n+1)}{\sqrt{2 q_{0}}}\left(\|w\|_{C_{a, b}^{\prime}}+|q|\left\|x_{0}\right\|_{C_{a, b}^{\prime}}\right)\|a\|_{C_{a, b}^{\prime}}\right\} d|f|(w) \\
= & \left(k_{n}\left(q_{0} ; x_{0}\right)\right)^{|q|} \int_{C_{a, b}^{\prime}[0, T]} k_{n}\left(q_{0} ; w\right) d|f|(w) \\
< & +\infty .
\end{aligned}
$$

Thus we see that $F \in \mathcal{F}_{n}^{q_{0}}$ implies $F^{*} \in \mathcal{F}_{n}^{q_{0}}$.

For notational convenience, we will write

$$
\vec{\eta}+\left(\vec{g}, x_{0}\right)_{C_{a, b}^{\prime}}=\left(\eta_{1}+\left(g_{1}, x_{0}\right)_{C_{a, b}^{\prime}}, \ldots, \eta_{n}+\left(g_{n}, x_{0}\right)_{C_{a, b}^{\prime}}\right)
$$

for $\vec{\eta} \in \mathbb{R}^{n}, x_{0} \in C_{a, b}^{\prime}[0, T]$ and $\left\{g_{1}, \ldots, g_{n}\right\} \subset C_{a, b}^{\prime}[0, T]$.

In Theorem 5.8, we obtain a translation theorem for CGFFT of functionals in $\mathcal{F}\left(C_{a, b}[0, T]\right)$.

Theorem 5.8. Let $X, q_{0}$ and $F$ be as in Theorem 5.3. Let $x_{0} \in C_{a, b}^{\prime}[0, T]$. Then for all real $q$ with $|q|>q_{0}$,

$$
\begin{aligned}
& T_{q}^{(p)}(F \mid X)\left(y+x_{0}, \vec{\eta}\right) \\
= & \exp \left\{i q\left(x_{0}, y\right)^{\sim}+i q\left(x_{0},[\vec{\eta}]+\left[x_{0}\right]\right)_{C_{a, b}^{\prime}}+\frac{i q}{2} B\left(p\left(x_{0}\right)\right)+i q(-i q)^{-1 / 2} A\left(p\left(x_{0}\right)\right)\right\} \\
& \times T_{q}^{(p)}\left(F^{*} \mid X\right)\left(y, \vec{\eta}+\left(\vec{g}, x_{0}\right)_{C_{a, b}^{\prime}}\right)
\end{aligned}
$$

for s-a.e. $y \in C_{a, b}[0, T]$, where $F^{*}$ is given by the equation (5.12).

Proof. From (ii) of Remark 5.7 we know that $F^{*} \in \mathcal{F}_{n}^{q_{0}}$ and so $T_{q}^{(p)}\left(F^{*} \mid X\right)$ exists for all $p \in[1,2]$ by Theorem 5.3. Thus we need only to verify the equality in equation (5.14).

We first note that for all $w \in C_{a, b}^{\prime}[0, T]$ and all $q \in \mathbb{R}$ with $|q|>q_{0}$,

$$
\begin{gathered}
\left(w, x_{0}\right)^{\sim}=\int_{0}^{T} D_{t} w d x_{0}(t)=\int_{0}^{T} D_{t} w D_{t} x_{0} d b(t)=\left(w, x_{0}\right)_{C_{a, b}^{\prime}} \\
{\left[\vec{\eta}+\left(\vec{g}, x_{0}\right)_{C_{a, b}^{\prime}}\right]=[\vec{\eta}]+\left[x_{0}\right]} \\
A\left(p\left(w-q x_{0}\right)\right)=A(p(w))-q A\left(p\left(x_{0}\right)\right)
\end{gathered}
$$


and

$$
\begin{aligned}
& B\left(p\left(w-q x_{0}\right)\right) \\
= & B(p(w))+q^{2} B\left(p\left(x_{0}\right)\right)-2 q\left(w, x_{0}\right)_{C_{a, b}^{\prime}}+2 q\left(w,\left[x_{0}\right]\right)_{C_{a, b}^{\prime}} .
\end{aligned}
$$

Next using (5.6) with $F$ and $\vec{\eta}$ replaced with $F^{*}$ and $\vec{\eta}+\left(\vec{g}, x_{0}\right)_{C_{a, b}^{\prime}}$ respectively, (5.16), (5.5) with $\lambda$ and $w$ replaced with $-i q$ and $h=w-q x_{0}$ respectively, (5.17), (5.18), (5.15) and (5.6), we have that for s-a.e. $y \in C_{a, b}[0, T]$ and $\vec{\eta} \in \mathbb{R}^{n}$,

$$
\begin{aligned}
& T_{q}^{(p)}\left(F^{*} \mid X\right)\left(y, \vec{\eta}+\left(\vec{g}, x_{0}\right)_{C_{a, b}^{\prime}}\right) \\
= & \int_{C_{a, b}^{\prime}[0, T]} \exp \left\{i(h, y)^{\sim}+i\left(h,[\vec{\eta}]+\left[x_{0}\right]\right)_{C_{a, b}^{\prime}}\right\} \Psi(-i q ; p(h)) d f_{q x_{0}}^{*}(h) \\
= & \int_{C_{a, b}^{\prime}[0, T]} \exp \left\{i(w, y)^{\sim}-i q\left(x_{0}, y\right)^{\sim}+i(w,[\vec{\eta}])_{C_{a, b}^{\prime}}+i\left(w,\left[x_{0}\right]\right)_{C_{a, b}^{\prime}}\right. \\
& \left.\quad-i q\left(x_{0},[\vec{\eta}]+\left[x_{0}\right]\right)_{C_{a, b}^{\prime}}\right\} \\
& \times \exp \left\{i(-i q)^{-1 / 2}\left(A(p(w))-q A\left(p\left(x_{0}\right)\right)\right)\right. \\
= & \exp \left\{-i q\left(x_{0}, y\right)^{\sim}-i q\left(x_{0},[\vec{\eta}]+\left[x_{0}\right]\right)_{C_{a, b}^{\prime}}-\frac{i q}{2} B\left(p\left(x_{0}\right)\right)-i q(-i q)^{-1 / 2} A\left(p\left(x_{0}\right)\right)\right\} \\
& \times \int_{C_{a, b}^{\prime}[0, T]} \exp \left\{i\left(w, y+x_{0}\right)^{\sim}+i(w,[\eta])_{C_{a, b}^{\prime}}\right\} \Psi(-i q ; p(w)) d f(w) \\
= & \exp \left\{-i q\left(x_{0}, y\right)^{\sim}-i q\left(x_{0},[\vec{\eta}]+\left[x_{0}\right]\right)_{C_{a, b}^{\prime}}-\frac{i q}{2} B\left(p\left(x_{0}\right)\right)-i q(-i q)^{-1 / 2} A\left(p\left(x_{0}\right)\right)\right\} \\
& \times T_{q}^{(p)}(F \mid X)\left(y+x_{0}, \vec{\eta}\right) .
\end{aligned}
$$

Equation (5.14) follows from the equation (5.19).

Corollary 5.9. Let $X, q_{0}$ and $F$ be as in Theorem 5.3. Let $x_{0} \in C_{a, b}^{\prime}[0, T]$. Then for all real $q$ with $|q|>q_{0}$,

$$
\begin{aligned}
& E^{a n f_{q}}(F \mid X)\left(y+x_{0}, \vec{\eta}\right) \\
= & \exp \left\{i q\left(x_{0},[\vec{\eta}]+\left[x_{0}\right]\right)_{C_{a, b}^{\prime}}+\frac{i q}{2} B\left(p\left(x_{0}\right)\right)+i q(-i q)^{-1 / 2} A\left(p\left(x_{0}\right)\right)\right\} \\
& \times E^{\operatorname{anf}_{q}\left(F^{*} \mid X\right)\left(\vec{\eta}+\left(\vec{g}, x_{0}\right)_{C_{a, b}^{\prime}}\right)}
\end{aligned}
$$

for s-a.e. $y \in C_{a, b}[0, T]$, where $F^{*}$ is given by the equation (5.12). 


\section{Examples}

In this section we present several important functionals to apply our results in previous sections.

Let $S: C_{a, b}^{\prime}[0, T] \rightarrow C_{a, b}^{\prime}[0, T]$ be the linear operator defined by

$$
S w(t)=\int_{0}^{t} w(s) d b(s) .
$$

Then the adjoint operator $S^{*}$ of $S$ is given by

$$
S^{*} w(t)=\int_{0}^{t}(w(T)-w(s)) d b(s) .
$$

Using an integration by parts formula, we see that

$$
\left(S^{*} b, x\right)^{\sim}=\int_{0}^{T} x(t) d b(t)
$$

Example 6.1. The functional

$$
F_{1}(x)=\exp \left\{i \int_{0}^{T} x(t) d b(s)\right\}
$$

is a functional under our consideration because

$$
F_{1}(x)=\exp \left\{i\left(S^{*} b, x\right)^{\sim}\right\}=\int_{C_{a, b}^{\prime}[0, T]} \exp \left\{i(w, x)^{\sim}\right\} d \delta_{1}(w),
$$

where $\delta_{1}$ is the Dirac measure concentrated at $S^{*} b$ in $C_{a, b}^{\prime}[0, T]$. Obviously, $F_{1}$ is an element of $\mathcal{F}_{n}^{q_{0}}$ for all $q_{0}>0$.

Example 6.2. Let $\mathcal{M}(\mathbb{R})$ be the class of complex-valued countably additive measures on $\mathcal{B}(\mathbb{R})$, the Borel class of $\mathbb{R}$. For $\nu \in \mathcal{M}(\mathbb{R})$, the Fourier transform $\widehat{\nu}$ of $\nu$ is a complex-valued function defined on $\mathbb{R}$ by the formula

$$
\widehat{\nu}(u)=\int_{\mathbb{R}} \exp \{i u v\} d \nu(v) .
$$

Given $m$ and $\sigma^{2}$ in $\mathbb{R}$ with $\sigma^{2}>0$, let $\nu_{m, \sigma^{2}}$ be the Gaussian measure given by

$$
\nu_{m, \sigma^{2}}(B)=\left(2 \pi \sigma^{2}\right)^{-1 / 2} \int_{B} \exp \left\{-\frac{(v-m)^{2}}{2 \sigma^{2}}\right\} d v, \quad B \in \mathcal{B}(\mathbb{R}) .
$$

Then $\nu_{m, \sigma^{2}} \in \mathcal{M}(\mathbb{R})$ and

$$
\widehat{\nu_{m, \sigma^{2}}}(u)=\int_{\mathbb{R}} \exp \{i u v\} d \nu_{m, \sigma^{2}}(v)=\exp \left\{-\frac{1}{2} \sigma^{2} u^{2}+i m u\right\} .
$$

Let $h \in C_{a, b}^{\prime}[0, T]$ and let $\nu \in \mathcal{M}(\mathbb{R})$. Define $F_{2}: C_{a, b}[0, T] \rightarrow \mathbb{C}$ by

$$
\begin{aligned}
F_{2}(x) & =\widehat{\nu_{m, \sigma^{2}}}\left((h, x)^{\sim}\right) \\
& =\exp \left\{-\frac{1}{2} \sigma^{2}\left[(h, x)^{\sim}\right]^{2}+i m(h, x)^{\sim}\right\} .
\end{aligned}
$$


Define a function $\phi: \mathbb{R} \rightarrow C_{a, b}^{\prime}[0, T]$ by $\phi(v)=v h$ and let $f_{2}=\nu_{m, \sigma^{2}} \circ \phi^{-1}$. It is quite clear that $f_{2}$ is in $\mathcal{M}\left(C_{a, b}^{\prime}[0, T]\right)$ and is supported by $[h]$, the subspace of $C_{a, b}^{\prime}[0, T]$ spanned by $\{h\}$. Now for s-a.e. $x \in C_{a, b}[0, T]$,

$$
\begin{aligned}
& \int_{C_{a, b}^{\prime}[0, T]} \exp \left\{i(w, x)^{\sim}\right\} d f_{2}(w) \\
= & \int_{C_{a, b}^{\prime}[0, T]} \exp \left\{i(w, x)^{\sim}\right\} d\left(\nu_{m, \sigma^{2}} \circ \phi^{-1}\right)(w) \\
= & \int_{\mathbb{R}} \exp \left\{i(\phi(v), x)^{\sim}\right\} d \nu_{m, \sigma^{2}}(v) \\
= & \int_{\mathbb{R}} \exp \left\{i(h, x)^{\sim} v\right\} d \nu_{m, \sigma^{2}}(v) \\
= & F_{2}(x) .
\end{aligned}
$$

Thus $F_{2}$ is an element of $\mathcal{F}\left(C_{a, b}[0, T]\right)$. Moreover $F \in \mathcal{F}_{n}^{q_{0}}$ for all $q_{0}>0$, because

$$
\begin{aligned}
& \int_{C_{a, b}^{\prime}[0, T]} k_{n}\left(q_{0} ; w\right) d\left|f_{2}\right|(w) \\
= & \int_{C_{a, b}^{\prime}[0, T]} k_{n}\left(q_{0} ; w\right) d f_{2}(w) \\
= & \int_{\mathbb{R}} \exp \left\{(n+1)\left(2 q_{0}\right)^{-1 / 2}\|v h\|_{C_{a, b}^{\prime}}\|a\|_{C_{a, b}^{\prime}}\right\} d \nu_{m, \sigma^{2}}(v) \\
= & \int_{\mathbb{R}} \exp \left\{-\frac{(v-m)^{2}}{2 \sigma^{2}}+(n+1)\left(2 q_{0}\right)^{-1 / 2}\|h\|_{C_{a, b}^{\prime}}\|a\|_{C_{a, b}^{\prime}}|v|\right\} d v \\
< & +\infty .
\end{aligned}
$$

Thus we can apply the results in previous sections to the functional $F_{2}$.

In particular, if we choose $h=S^{*} b, m=0$ and $\sigma^{2}=2$ in the last expression of $(6.2)$, then we have

$$
F_{3}(x)=\exp \left\{-\left(\int_{0}^{T} x(t) d b(t)\right)^{2}\right\} .
$$

The functionals given by (6.1) and (6.3) are interpreted as the potential energy in quantum mechanics.

\section{References}

[1] R. H. Cameron and D. A. Storvick, Some Banach algebras of analytic Feynman integrable functionals, Analytic Functions, Kozubnik 1979 (Proc. Seventh Conf., Kozubnik, 1979), pp. 18-67, Lecture Notes in Math., 798, Springer, Berlin-New York, 1980.

[2] S. J. Chang and D. M. Chung, Conditional function space integrals with applications, Rocky Mountain J. Math. 26 (1996), no. 1, 37-62. 
[3] S. J. Chang and D. Skoug, Parts formulas involving conditional Feynman integrals, Bull. Austral. Math. Soc. 65 (2002), no. 3, 353-369.

[4] _ Generalized Fourier-Feynman transforms and a first variation on function space, Integral Transforms Spec. Funct. 14 (2003), no. 5, 375-393.

[5] S. J. Chang and J. G. Choi, Conditional generalized Fourier-Feynman transform and conditional convolution product on a Banach algebra, Bull. Korean Math. Soc. 41 (2004), no. 1, 73-93.

[6] _ Multiple $L_{p}$ analytic generalized Fourier-Feynman transform on the Banach algebra, Commun. Korean Math. Soc. 19 (2004), no. 1, 93-111.

[7] S. J. Chang, J. G. Choi, and D. Skoug, Integration by parts formulas involving generalized Fourier-Feynman transforms on function space, Trans. Amer. Math. Soc. 355 (2003), no. 7, 2925-2948.

[8] , Parts formulas involving conditional generalized Feynman integrals and conditional generalized Fourier-Feynman transforms on function space, Integral Transforms Spec. Funct. 15 (2004), no. 6, 491-512.

[9] _ Evaluation formulas for conditional function space integrals. I, Stoch. Anal. Appl. 25 (2007), no. 1, 141-168.

[10] _ Simple formulas for conditional function space integrals and applications, Integration: Mathematical Theory and Applications 1 (2008), 1-20.

[11] S. J. Chang, H. S. Chung, and D. Skoug, Integral transforms of functionals in $L^{2}\left(C_{a, b}[0, T]\right)$, J. Fourier Anal. Appl. 15 (2009), no. 4, 441-462.

[12] S. J. Chang, J. G. Choi, and S. D. Lee, A Fresnel type class on function space, J. Korea Soc. Math. Educ. Ser. B Pure Appl. Math. 16 (2009), no. 1, 107-119.

[13] D. M. Chung, Conditional analytic Feynman integrals on abstract Wiener spaces, Proc. Amer. Math. Soc. 112 (1991), no. 2, 479-488.

[14] D. M. Chung and D. L. Skoug, Conditional analytic Feynman integrals and a related Schrödinger integral equation, SIAM J. Math. Anal. 20 (1989), no. 4, 950-965.

[15] G. W. Johnson and D. L. Skoug, Notes on the Feynman integral. III. The Schroedinger equation, Pacific J. Math. 105 (1983), no. 2, 321-358.

[16] G. W. Johnson and M. L. Lapidus, The Feynman Integral and Feynman's Operational Calculus, Clarendon Press, Oxford, 2000.

[17] C. Park and D. Skoug, A simple formula for conditional Wiener integrals with applications, Pacific J. Math. 135 (1988), no. 2, 381-394.

[18] _ Conditional Fourier-Feynman transforms and conditional convolution products, J. Korean Math. Soc. 38 (2001), no. 1, 61-76.

[19] J. Yeh, Singularity of Gaussian measures on function spaces induced by Brownian motion processes with non-stationary increments, Illinois J. Math. 15 (1971), 37-46.

[20] _ Stochastic Processes and the Wiener Integral, Marcel Dekker, Inc., New York, 1973

[21] _ Inversion of conditional Wiener integrals, Pacific J. Math. 59 (1975), no. 2, $623-638$.

Department of Mathematics

DANKOOK UNIVERSITY

Cheonan 330-714, Korea

E-mail address: sejchang@dankook.ac.kr 